\title{
PARITY CONSERVATION IN ATOMS: TESTING LAPORTE'S RULE
}

\author{
R. R, LEWIS ${ }^{\dagger}$
}

University of Michigan, Ann Arbor, MI

\section{ABSTRACT}

There are two independent reasons to expect the existence of a new type of weak interaction, involving elastic scattering of electrons and nucleons, $e+N \rightarrow e+N$. One is the successful development of unified theories of weak and electromagnetic interactions, based on isospin multiplets of leptons, hadrons and vector mesons. The other is the experimental discovery of the elastic scaftering of high energy neutrinos from nucleons, $v+N \rightarrow v+N$. Together, these developments provide strong motivation to the search for weak electron-nucleon interactions in ordinary atoms, through a breakdown of Laporte's rule. I will present a review of recent developments in this field, including a qualitative discussion of this type of weak interaction and a short summary of the experiments in progress. I will also give a more detailed discussion of one particular experiment at Michigan, involving microwave transitions in a metastable hydrogen beam.

\section{At present, there is no experimental evidence of a failure of Laporte's} rule. The most recent data from optical activity in bismuth vapor, has probably ruled out the gauge model of Weinberg-Salam.

\section{A. BRIEF HISTORY OF PARITY IN ATOMS}

In 1924, Laportel analyzed the atomic spectrum of i ron and found, among other conclusions, that the states could be classified as either 'primed' or 'unprimed'. Three years later, Wigner ${ }^{2}$ showed that this classification followed from symmetry under spatial inversions, with 'primed' or 'unprimed' states being even or odd under inversion. Only thirty years later was it discovered by Lee and $\mathrm{Yang}^{3}$ that this symmetry was broken in the weak interaction governing beta decay. Since the classic weak interactions do not influence atomic states, to order $G$, there was no reason to expect a breakdown of Laporte's rule. Only very recently, through technical advances such as the development of the laser, has it been possible to test parity conservation in atoms to terms of order $G$. Earlier parity experiments ${ }^{4}$ were only sensitive to interactions several orders of magnitude stronger than $G$. In this article, I will review recent developments concerning this subject. The main conclusion will be that there are good theoretical reasons to expect parity nonconservation in atoms, but still no experimental evidence for this. Thus Laporte's rule, after 53 years, has survived its first test at the level of $G$. An extensive search for parity nonconservation is underway in several laboratories.

An out?ine of this paper includes three main topics:

1) a qualitative discussion of the form of the parity nonconserving potential, and its consequences in atoms.

2) a brief summary of current parity experiments.

3) a more detailed discussion of a particular microwave experiment in metastable hydrogen, in progress at the Univ. of Michigan.

Some discussion of the theory is necessary to motivate the design of experiments, and to provide a framework for quoting experimental results. I have decided to $1 \mathrm{imit}$ this discussion to the form predicted for the non-relativistie potential, and to move as quickly as possible into a review of the experimental situation. At present, there are more theoretical models than experimental results, so the important future developments will probably be on the experimental side. To counteract the necessarily superficial review of the experiments, 1 have chosen to analyse just one in some detail. Since 1 am directly involved in one of the competing groups, you will have to excuse my choosing an experiment which

\footnotetext{
TResearch supported by the National Science Foundation under grant PHY76-02855
} 
I know well, and which we think holds the most promise for significant results.

The paper will conclude with some very brief remarks on the implications of these experiments and a trick slide giving a do-it-yourself parity experiment.

\section{B. QUALITATIVE DISCUSSION OF WEAK INTERACTION THEORY}

Our understanding of weak interactions has undergone a steady but remarkably slow progress during the 40 years since the theory was first formulated by Fermi. He described them as a local interaction between pairs of spin $1 / 2$ particles,

$$
H=G \int d x\left(\psi_{1} O \psi_{2}\right)\left(\psi_{3} O \psi_{4}\right)+h . c .
$$

characterised by a single constant

$$
G=1.4 \times 10^{-49} \mathrm{erg}-\mathrm{cm}^{3}=2.2 \times 10^{-14} \mathrm{e}^{2} \mathrm{a}_{0}^{2} .
$$

This constant appears universally in interactions of this form; its very small value accounts for the slow progress.

We have learned to think of such interactions in terms of currents of spin $1 / 2$ particles, interacting with intermediate vector mesons (Fig. 1). The small

(a)

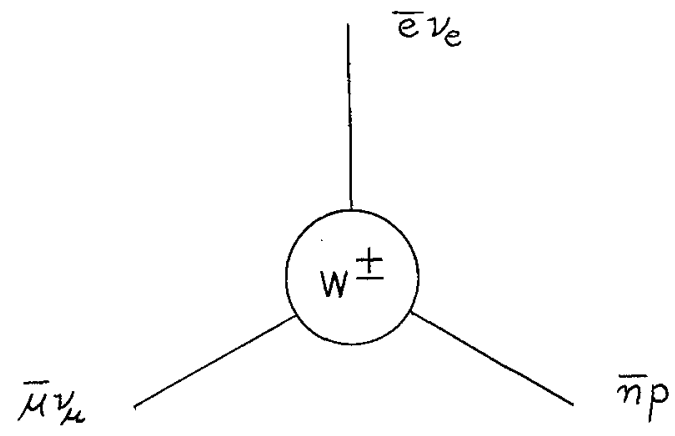

(b)

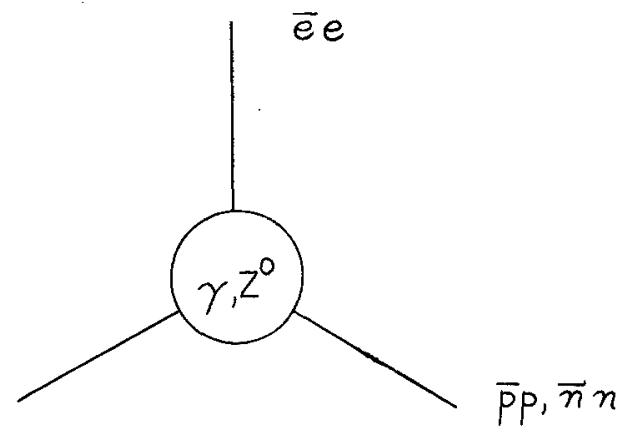

Figure 1 (a) Charged Currents coupled to $W^{ \pm}$vector mesons.

(b) Neutral Currents coupled to $\boldsymbol{\gamma}, z^{\circ}$ vector mesons. 
value of $G$ and the short range of interaction are related to the large mass of these (unobserved) particles. The classical weak interactions ${ }^{3}$ (beta decay, muon decay, muon capture) involve charged currents, interacting via exchange of charged $W \pm$ vector mesons (Fig. la). We label these currents by the charge transfer, and the charge of the exchanged meson, not by the net charge of the spin $1 / 2$ particles.

The development of unified theories of electromagnetism and weak interactions ${ }^{5}$ has arisen from the idea of a gauge symmetry which assigns the leptons, hadrons and vector mesons to isospin multiplets. In addition to the electromagnetic interactions, which involve neutral lepton and hadron currents interacting via a massless photon $\gamma$, it is natural to introduce another very massive neutral meson $Z^{\circ}$ (Fig. $\mathrm{Ib}$ ). The $Z^{\circ}$ meson would complete an isospin triplet with $W^{ \pm}$, and would $i \mathrm{mp} l y$ the existence of weak interactions between neutral pairs such as $\bar{v}, \bar{e}, \bar{p} p$ and $\bar{n} n$.

There is nothing exotic about such interactions. They involve elastic reactions such as $v+N \rightarrow v+N$ and $e+N \rightarrow e+N$, between well known particles. They were considered many years ago among the universal weak interactions which conserve baryon and lepton number. ${ }^{6}$ There was no reason to exclude them, but no evidence for their existence, due largely to the difficulty of observing such reactions in a background of stronger processes. In 1973, the first experimental evidence ${ }^{7}$ for neutral currents of neutrinos and nucleons came with the discovery of elastic scattering $v+p \rightarrow v+p$ and inelastic reactions $v+N \rightarrow v+N+\pi$. Subsequently, there has been confirmation of these processes, and further study of their isospin dependence and V,A structure. ${ }^{8}$ Elastic scattering $v+e \rightarrow v+e$ has also been observed, 9 confirming the basic validity of Fig. (1b).

\section{Parity Nonconserving Atomic Forces}

Gauge theories provide some general insight into the nature of weak interactions between electrons and nucleons, but no single choice of the gauge group or of the multiplet assignments is indicated. A useful way to proceed with a general analysis of these weak interactions in ordinary atoms is to concentrate on the form of the non-relativistic electron-nucleon potential which would result, assuming only certain broad characteristics of the theory and leaving the parameters free for comparison with experiment. The parity conserving (PC) part of this interaction will be an unobservable correction to electromagnetic forces; only the parity non-conserving (PNC) part will be retained.

Since the potential has a range of order $\pi / M_{Z} c$, much shorter than atomic radii, the potential will be made proportional to a delta function in the relative coordinate, $\delta(r)$. And since atoms are non-relativistic systems, we will expand the potential in powers of $p$, retaining only the leading terms in the electron velocity $\mathrm{p} / \mathrm{m}$ and ignoring the nucleon velocity, $\mathrm{p} / \mathrm{M}$. As a final simplifying assumption, we will retain only the T-invariant potentials. There is already good experimental evidence ${ }^{10}$ that P-odd, T-odd interactions in atoms are absent to a level of order $10^{-3} \mathrm{G}$.

Under these assumptions, there are only three possible forms which the electron-proton potential can have, each linear in $\mathrm{p} / \mathrm{m}$,

$$
\begin{aligned}
& v_{1}=-c_{1 p} \frac{G}{\sqrt{8 m c}}\left\{\sigma_{e} \cdot p \delta(r)+h \cdot c \cdot\right\} \\
& \left.v_{2}=+c_{2 p} \frac{G}{\sqrt{8 m c}}\left\{\sigma_{p} \cdot p+i \sigma_{p} \times \sigma_{e} \cdot p\right) \delta(r)+h . c .\right\} \\
& v_{3}=+c_{3 p} \frac{G}{\sqrt{8 m c}}\left\{i \sigma_{p} \times \sigma_{e} \cdot p \delta(r)+h \cdot c .\right\}
\end{aligned}
$$


and three similar terms for the electron-neutron potential. We see that in each case the potential depends in a specific way on the spins and momenta, and has a single real constant $C$ to be determined. Since we have removed appropriate dimensional factors, the six dimensionless constants $c_{1 p} \ldots c_{3 n}$ are presumed to be of order unity or smaller. We notice that $V_{1}$ is independent of the nucleon spin, but $V_{2}$ and $V_{3}$ are not.

The origin of these potentials can be traced back to the relativistic form of the four-fermion interaction, 13

$$
\begin{aligned}
H=\frac{G}{\sqrt{2}} \int d x\left[c_{1 p}\left(\bar{e} \gamma_{\mu} \gamma_{5} e\right)\left(\bar{p} \gamma_{\mu} p\right)+c_{2 p}\left(\bar{e} \gamma_{\mu} e\right)\left(\bar{p} \gamma_{\mu} \gamma_{5} p\right)\right. \\
\left.+i c_{3 p} \frac{1}{2 m}\left\{\frac{\partial}{\partial x_{\nu}}\left(\bar{e} \sigma_{\nu \mu} e\right)\right\}\left(\bar{p} \gamma_{\mu} \gamma_{5} p\right)\right] .
\end{aligned}
$$

Looking at the nucleon factors, and using the familiar nomenclature of beta-decay theory, the $\mathrm{C}_{1}$ term is the neutral analogue of a Fermi interaction, $\mathrm{c}_{2}$ is the analogue of a Gamow-Teller interaction and $C_{3}$ is the analogue of the weak magnetism contribution, involving the electron anomalous magnetic moment.

These couplings can be traced back further to specific gauge models, which are defined in terms of quarks and vector mesons rather than the physical nucleon. The range of possible values of the four parameters $C_{1 p}, C_{1 n}, C_{2 p}, C_{2 n}$ can be illustrated by Fig. 2, taken from a recent publication? ${ }^{2}$ Ip, Although there are a large number of gauge models, each model is characterised by at most one parameter, $K$, which expresses the freedom to adjust the overall strength of the neutral currents relative to the charged currents. This implies that one or two measurements are sufficient to contradict any given model. Notice that there are gauge models which predict zero for each $c$, and thus parity conservation in atoms.

Most gauge models imply that the weak electron current is the same as the electromagnetic current, and that the ratio of the 'weak magnetism' term $C_{3}$ to the 'weak charge' term $C_{2}$ is the same as the measured anomalous moment of the electron in Bohr magnetons,

$$
c_{3 p} / c_{2 p} \cong \alpha / 2 \pi
$$

It may be possible to test this specific prediction in hydrogen, but otherwise it is probably wise to drop $C_{3}$ and concentrate on the other constants.

\section{Parity Mixing in Atomic States}

The advantages in construction of a potential are that we can immediately read off several general features of its matrix elements, and provide detailed results for atoms with known wavefunctions. For example, the order of magnitude of the weak matrix element in a typical atomic state can be estimated. Since $<p / m c>N a$ and $<\delta(r)>\sim 1 / a_{0}^{3}$,

$$
\left\langle V>\sim G \alpha / a_{0}^{3} \simeq 10^{-14} \times 10^{-2} \times 1 \text { a.u. }=10^{-16}\right. \text { a.u. }
$$

We might compare this with a typical level separation $\Delta E_{2} 10^{-1}$ a.u., which implies that the parity mixtures $F$ are of order

$$
F_{\text {typical }}=\frac{\langle V\rangle}{\Delta E} \sim 10^{-15}
$$


$\mathrm{T}_{3 \mathrm{R}}^{\mathrm{e}}=0$

$$
\mathrm{T}_{3 \mathrm{R}=}^{\mathrm{U}}=0 \quad \mathrm{~T}_{3 \mathrm{R}}^{\mathrm{d}}=0
$$

$\mathrm{T}_{3 \mathrm{R}}^{\mathrm{u}}=1 / 2 \mathrm{~T}_{3 \mathrm{R}}^{\mathrm{d}}=0$

$$
\begin{aligned}
& C_{1 p}=K\left(3-4 \sin ^{2} \theta\right) / 2 \\
& C_{1 n}=0 \\
& C_{2 p}=K\left(1-4 \sin ^{2} \theta\right)(D-F) / 2 \\
& C_{2 p}=-K\left(1-4 \sin ^{2} \theta\right) F
\end{aligned}
$$

$$
\begin{aligned}
& C_{1 p}=K\left(1-4 \sin ^{2} \theta\right) / 2 \\
& C_{1 n}=-K / 2 \\
& C_{2 p}=K\left(1-4 \sin ^{2} \theta\right)(F+D) / 2 \\
& C_{2 n}=-K\left(1-4 \sin ^{2} \theta\right)(F+D) 2
\end{aligned}
$$$$
T_{3 R}^{e}=-1 / 2
$$

$$
\begin{aligned}
& C_{I p}=0 \\
& C_{I n}=0 \\
& C_{2 p}=K\left(2-4 \sin ^{2} \theta\right)(F+D) / 2 \\
& C_{2 n}=-K\left(2-4 \sin ^{2} \theta\right)(F+D) / 2
\end{aligned}
$$

$$
\begin{aligned}
\mathrm{T}_{3 R}^{\mathrm{U}}=0 \quad \mathrm{~T}_{3 \mathrm{R}}^{2}=-1 / 2 \quad \mathrm{C}_{1 \mathrm{p}} & =-2 \mathrm{~K} \sin ^{2} \theta \\
\mathrm{C}_{1 \mathrm{n}} & =-3 \mathrm{~K} / 2 \\
\mathrm{C}_{2 \mathrm{p}} & =\mathrm{K}\left(1-4 \sin ^{2} \theta\right) \mathrm{F} \\
\mathrm{C}_{2 \mathrm{n}} & =\mathrm{K}\left(1-4 \sin ^{2} \theta\right)(\mathrm{F}-\mathrm{D}) / 2
\end{aligned}
$$

$$
\begin{aligned}
& c_{1 p}=0 \\
& c_{1 n}=0 \\
& C_{2 p}=K\left(2-4 \sin ^{2} \theta\right)(D-F) / 2 \\
& C_{2 n}=-K\left(2-4 \sin ^{2} \theta\right) F
\end{aligned}
$$

$\mathrm{C}_{1 \mathrm{p}}=0$

$\mathrm{C}_{1 \mathrm{n}}=0$

$\mathrm{C}_{2 \mathrm{p}}=\mathrm{k}\left(2-4 \sin ^{2} \theta\right) \mathrm{F}$

$C_{2 n}=K\left(2-4 \sin ^{2} \theta\right)(F-D) / 2$

$$
\begin{aligned}
& \mathrm{C}_{1 \mathrm{p}}=0 \\
& \mathrm{C}_{1 \mathrm{n}}=0 \\
& \mathrm{C}_{2 \mathrm{p}}=0 \\
& \mathrm{C}_{2 \mathrm{n}}=0 \\
& \mathrm{~T}_{3 \mathrm{R}}^{\mathrm{u}}=1 / 2 \quad \mathrm{~T}_{3 \mathrm{R}}^{\mathrm{d}}=-1 / 2 \quad \mathrm{C}_{1 \mathrm{p}}=\mathrm{K}\left(2-4 \sin ^{2} \theta\right) / 2 \\
& \mathrm{C}_{1 \mathrm{n}}=-\mathrm{K} \\
& \mathrm{C}_{2 \mathrm{p}}=0 \\
& \mathrm{C}_{2 \mathrm{n}}=0
\end{aligned}
$$

Figure 2. Some typical examples of the values of $C_{1}$ and $C_{2}$ for various gauge models, reprinted by permission from Cahn and Kane (ref. 12). The various models are specified by the multiplet assignment of the quarks and the electron. The quantities $\theta, F, D$ should be considered as already known. 
This number is hopelessly small, forcing us to look for favorable situations which would show values larger than typical.

In hydrogen the relevant levels, with the same $F$ but opposite parity, are much closer than 'typical'. In the $n=2$ shell, the $25-2 P$ separation (Lamb shift) is $S=10^{3} \mathrm{MHz}$ and so the parity mixture is

$$
F_{H} \simeq \frac{10^{-2} \mathrm{~Hz}}{10^{+9} \mathrm{~Hz}} \simeq 10^{-11}
$$

All the matrix elements of $V_{1}, V_{2}, V_{3}$ can be calculated to high accuracy, and give parity mixtures of this order for hydrogen in zero field. ${ }^{13}$ Hydrogen (or deuter$i$ um) is equally sensitive to $c_{1 p}, c_{2 p}$ and $c_{3 p}$ (or $c_{1 p}+c_{1 n}, c_{2 p}+c_{2 n}, c_{3 p}+c_{3 n}$ ).

For hydrogenic ions, the complete dependence on $n, \ell, z$ can also be calculated in detail. Clearly the factors $p \delta(r)$ imply that $V$ mixes only $S$ and $P$ states, and that the matrix elements contain a factor $R_{S}(0) R_{p}^{\prime}(0)$ from the radial functions. It can be shown ${ }^{13}$ that all of the matrix elements of $v_{1}, v_{2}, v_{3}$ are proportional to the quantity $\bar{v}$,

$$
\bar{V}=\frac{3}{8 \pi \sqrt{2}}\left(G \frac{\hbar}{m c}\right) R_{n s}(0) R_{n p}^{\prime}(0)=\left(\frac{G \alpha}{a_{0}^{3}}\right) \frac{2^{4} \sqrt{n^{2}-1}}{\pi n^{4} \sqrt{8}}
$$

which increases rapidly with $Z$ and decreases with $n$. The remaining factors depend only on the coupling constants and the spins. There is a significant difference between the nuclear spinindependent potential $V_{1}$, which involves only the sum of the coupling constants

$$
C_{1} \text { (nucleus) }=Z C_{I p}+(A-Z) C_{I n}=\left(\frac{1}{2} A\right) C_{1}^{i s o s c a l a r}+\left(Z-\frac{A}{2}\right) C_{1}^{i s o v e c t o r ~}
$$

and the other potentials $V_{2}, V_{3}$ which also involve the sum of the nucleon spins. The isoscalar part of the coupling contributes equally to $C_{1 p}$ and $C_{1 n}$, and grows linearly with atomic number $A$ and is therefore enhanced. The isovector part of $C_{1}$, as well as $C_{2}, C_{3}$, remains of order unity, since both spins and isospins pair off in nuclei.

The dependence of $\bar{V}$ on $z, n$ is almost the same as the dependence of fine structure and Lamb shift separations, which also are proportional to $Z^{4} / \mathrm{n}^{3}$. Aside from the logarithmic factors in the Lamb shift, which slowly reduce the separation in heavier atoms, and aside from the linear growth of $C_{1}$ (isoscalar), hydrogenic ions will have the same parity mixture as the hydrogen atom (Eq. 8).

Finally we consider the parity mixture in low-lying states of heavy atoms. The energy separations are now 'typical', but the overlap of the valence electrons at the nucleus grow strongly with $Z$ due to the large Coulomb field and to the relativistic enhancement. The calculation now involves difficult problems of atomic structure, and has been studied using a generalization of the semi-empirical Fermi-Segre method. ${ }^{14}$ Their final result for the behavior of $\left\langle V_{1}\right\rangle$, including the growth of $C$ isoscalar, shows an enhancement of $\left\langle V_{1}\right\rangle$ larger than $z^{3}$ and a parity mixture in Cesium

$$
F_{1}\left(c_{s}\right) \cong 10^{-10}
$$

The mixture due to $V_{2}, V_{3}$ would be approximately two orders smaller.

To summarize, the supposition of parity nonconserving weak interactions between electrons and nucleons leads to parity mixtures which are at most of 
order $10^{-9}$ to $10^{-11}$. These numbers indicate the difficulty of observing weak interactions in atoms, and explain why Laporte's rule has survived so long.

\section{SUMMARY OF RECENT EXPERIMENTS}

With this general background, let us turn to a survey of recent experiments capable of detecting parity nonconservation of order $G$, or measuring $C$ of order unity. These experiments are of interest because they can provide a test of gauge theories, and because they utilize recent technological advances in lasers, ion sources, signal averaging, etc. The survey will not be logical, but chronological. In each case I will try to provide the names, places, dates, together with a brief description of the equipment and method. To simplify the discussion I will give the scalars and the psuedoscalars measured in each experiment, since they provide the best insight into the physical variables involved. The status and/or results of each experiment will also be given.

1) Bouchiat and Bouchiat ${ }^{14}$ (Paris) in 1974 proposed an experiment on the $6 \mathrm{~S}_{1 / 2} \rightarrow 7 \mathrm{~S}_{1 / 2}$ transition $(539 \mathrm{~nm})$ in cesium vapor. This transition is strongly forbidden, since it involves an Ml transition between shells. The relatively large parity mixture $\left(F \simeq 10^{-10}\right)$ and the large ratio of weak induced $E l$ to intrinsic $M\}\left(E I / M I \simeq 10^{+6}\right)$ could lead to PNC effects of order $\mathrm{F}(\mathrm{El} / \mathrm{MI}) \simeq 10^{-4}$. A simplified description of the experiment is indicated in Fig. 3a, showing apparatus for measuring the circular dichroism (absorption vs circular polarization) of the incident light, using a tunable dye laser. The Doppler broadened line width ( $\Delta \nu \sim 750 \mathrm{MHz}$ ) is sufficiently narrow to permit resolution of the hyperfine structure. To begin with a simplified version, we consider the absorption, which is proportional to the square of the transition amplitude consisting of the sum of $M I$ and weak-induced El amplitudes,

$$
\text { absorption } a\left|(M I)+F(E I)_{W}\right|^{2} \text {. }
$$

This rate depends only on the polarization vector $\hat{\varepsilon}$ and the propagation vector $\hat{k}$ of the incident light; the $|M l|^{2}$ terms depend on the scalar $(\hat{\varepsilon} \times \hat{k}) \cdot(\hat{\varepsilon} \times \hat{k})=\hat{\varepsilon} \cdot \hat{\varepsilon}^{*}$ independent of circular polarization. The (MI)-(EI) $W$ interference terms depend on the pseudoscalar $\mathrm{i}\left(\hat{\varepsilon} \times \hat{\varepsilon}^{*}\right) \cdot \hat{k}$, which is the helicity of the incident beam (projection of circular polarization $i \hat{\varepsilon} x^{*}$ on propagation vector). Thus, a dependence of the absorption on the circular polarization is evidence for parity nonconservation.

The actual experiment, described in a series of papers by $M$. Bouchiat and L. Pottier, Is is considerably more intricate. The signal is detected by fluorescence of the $7 \mathrm{~S} \rightarrow 6 \mathrm{P}$ transition $(1360 \mathrm{~nm}$ ), rather than by absorption (see Fig. $3 b)$. This introduces into the analysis two additional vectors $\hat{\varepsilon}_{2}$, v $\hat{k}_{2}$ from the emitted $1 \mathrm{ight}$, and $\mathbf{i n c r e a s e s}$ the number of scalars and psuedoscalars. 16 The fluorescence signal can also be perturbed by application of electric and magnetic fields. ${ }^{17}$ An electrostatic field between $10-1000 \mathrm{~V} / \mathrm{cm}$ adds a third amplitude to the process, a Stark-induced electric dipole amplitude (EI) s arising from parity mixing caused by the electric field. This amplitude can be accurately calculated, with an estimated error $\leq 15 \%$, and serves as a convenient reference for measuring $(M 1)$ and $(E 1)_{W}$.

The actual measurements began with observation of the $6 \mathrm{~S} \rightarrow 7 \mathrm{~S}$ transition using linearly polarized light and a strong electric field (E $\cong 1000 / \mathrm{cm}$ ). The scalars $(\hat{\varepsilon} \cdot \vec{E})^{2}$ and $(\hat{E} \times \vec{E}) \cdot(\hat{E} \times \vec{E})$ were measured, confirming the calculated values of $(E I)_{s}$. Next (MI) was measured relative to (El) sy detecting the circular polarization of the fluorescent light, with the incident light linearly polarized parallel to $E$. The angular distribution measured in this step was $i\left(\hat{\varepsilon}_{2} \times \hat{\varepsilon}_{2} *\right) \cdot\left(\hat{\varepsilon}_{1} \times \hat{k}_{1}\right)\left(\hat{\varepsilon}_{1} \cdot \vec{E}\right)$, which is a scalar arising through interference of $(E I)_{5}$ 
and (MI). Finally, the parity experiment was performed by measuring a pseudoscalar quantity involving the circular polarization of both initial and final photons, $\left(\hat{\varepsilon}_{2} \times \hat{\varepsilon}_{2}{ }^{*}\right) \cdot\left(\hat{\varepsilon}_{I} \times \hat{\varepsilon}_{1}{ }^{*}\right) \times \vec{E}$. This term arises from the interference of $(E I)_{W}$ and $(E I)_{5}$, and changes sign when either circular polarization is reversed. In the experiment, both circular polarization vectors were modulated, and the psuedoscalar term was identified with double lock-in detection. At $E=1000 \mathrm{~V} / \mathrm{cm}$, the predicted signal is a variation of about $4 \times 10^{-6}$ under reversal of either circular polarization. We note that the final experiment does not utilize the MI amplitude, which is irrelevant to the actual parity experiment.

The parity experiment could also be performed at zero electric field by detection of the interference of $(M I)$ and $(E 1)_{W}$, which gives a psuedoscalar $i\left(\hat{\varepsilon}_{2} \times \hat{\varepsilon}_{2} *\right) \cdot \hat{k}_{1}\left(\hat{\varepsilon}_{1} \cdot \hat{\varepsilon}_{1} *\right)$. This term would give a larger relative signal, of order $10^{-4}$, but a lower fluorescence yield and fewer modulations to suppress background.

The results of these experiments have been an interesting series of measurements on the forbidden $6 \mathrm{~S} \rightarrow 7 \mathrm{~S}$ transition but no evidence for parity nonconservation. The latest published report gives a null result which puts an upper limit on the weak induced electric dipole moment

$$
\mathrm{e}|<7 \mathrm{~S}| \mathrm{z}|6 \mathrm{~S}>|<2 \times 10^{-9} \mathrm{ea}_{\mathrm{o}}
$$

corresponding to an upper limit on $C_{1 p}$ and $C_{1 n}$

$$
\left(Z C_{1 p}+N C_{1 n}\right) / A<44
$$

These $1 \mathrm{imits}$ are about 100 times larger than predicted by gauge models.

There is a similar experiment on cesium in progress at Chicago (1977).

2) There has been significant Russian work on this topic, including a proposal to study the circular polarization of Lyman-alpha photons in the hydrogen atom $\left(25 \rightarrow\right.$ is transition) by Moskalev ${ }^{18}$ in 1974. There is also a study of the feasibility of measuring the optical activity of heavy metal vapors by Khriplovich ${ }^{19}$ in 1974. However, there is no mention of experiments actually in progress in Russia. 20

3) There are two similar laser experiments involving the rotation of polarized light (optical ac tivity) at resonance in bismuth vapor. The first, begun at Oxford by Sandars 21 in 1975, uses the transition $6 p^{3} s_{3 / 2} \rightarrow 6 p^{3}{ }^{2} D_{5 / 2}$ $(\lambda=648 \mathrm{~nm})$ between ground and second excited state. The other, begun at Washington by Fortson ${ }^{22}$ in 1975, uses the first excited state $S_{3 / 2} \rightarrow{ }^{2} D_{3 / 2}$ $(\lambda=878 \mathrm{~nm})$. Both experiments involve a measurement of the transmission of light through a bismuth cell placed between crossed linear polarizers, Fig. 4. There are no fields applied to the $\mathrm{Bi}$ sample, but an additional Faraday rotator with axial magnetic field is inserted in the optical path to provide modulation of the polarization.

The basic idea underlying both of these experiments can best be analyzed in terms of the index of refraction or forward scattering amplitude for light. If we include only the PC amplitude (MI), then the index of refraction can only depend on the scalars $\left(\hat{E}_{1} \times \hat{k}\right) \cdot\left(\hat{\varepsilon}_{2} \times \hat{k}\right)=\hat{\varepsilon}_{1} \cdot \hat{\varepsilon}_{2}$ and $\left(\hat{\varepsilon}_{1} \times \hat{k}\right) \times\left(\hat{\varepsilon}_{2} \times \hat{k}\right) \cdot B=\left(\hat{\varepsilon}_{1} \times \hat{\varepsilon}_{2} \cdot \hat{k}\right)(B \cdot \hat{k})$. Adding a small PNC amplitude (EI) $W$, the index of refraction can also contain the psuedoscalar term $\left(\hat{\varepsilon}_{1} \times \hat{\varepsilon}_{2} \cdot \hat{k}\right)$, from $(M 1)-(E 1)$ interference. Here $\hat{\varepsilon}_{1}$ is the polarization vector of the incident light (polarizer) and $\hat{\varepsilon}_{2}$ is the polarization vector of the transmitted light (analyzer). 
These three terms make three separate contributions to the amplitude of the light coming through crossed polarizers. The first term $\hat{\varepsilon}_{1} \cdot \hat{\varepsilon}_{2}$ would be zero for an ideal optical system, but is nonzero due to several systematic effects

(strains in windows, imperfect polarizer and analyzer, etc.). Expressed in terms of the angle of rotation of the plane of polarization, this term contributes a residual angle $\phi_{0} \simeq 10^{-4}$ radians, or a transmission $\left|\phi_{0}\right|^{2} \simeq 10^{-8}$. The second term $\left(\hat{\varepsilon}_{1} \times \hat{\varepsilon}_{2} \cdot \hat{k}\right)(\vec{B} \cdot \hat{k})$ contributes a variable rotation angle $\phi_{M}$ proportional to the magnetic field. The third term $\left(\hat{\varepsilon}_{1} \times \hat{\varepsilon}_{2} \cdot \hat{k}\right)$ gives the rotation due to weak interactions, $\phi_{W}$. The overall transmission

$$
T=\left|\phi_{0}+\phi_{W}+\phi_{M}\right|^{2}
$$
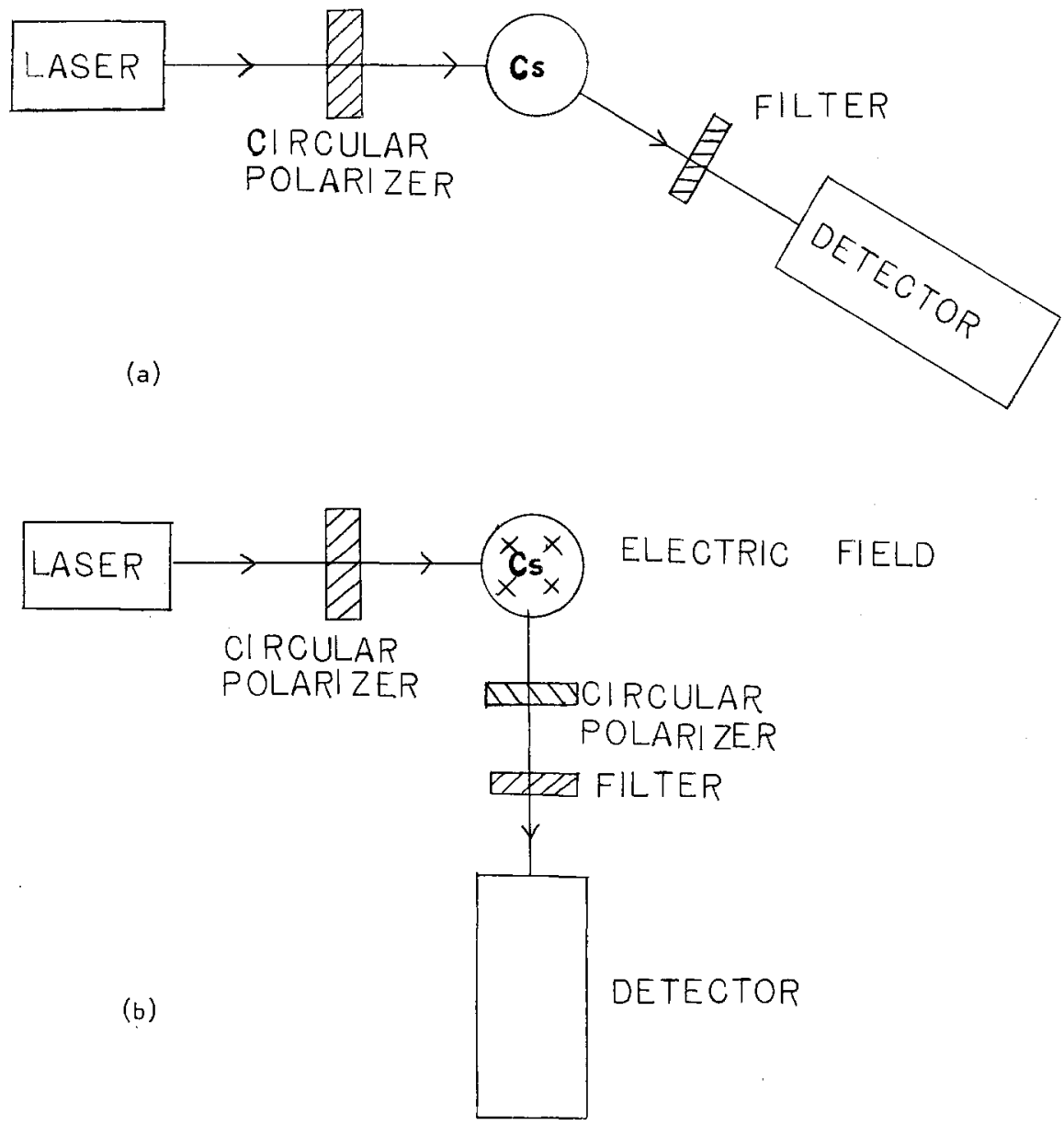

Figure 3. (a) Apparatus to measure circular dichroism in Cesium (b) Apparatus to measure perturbed resonance fluorescence 


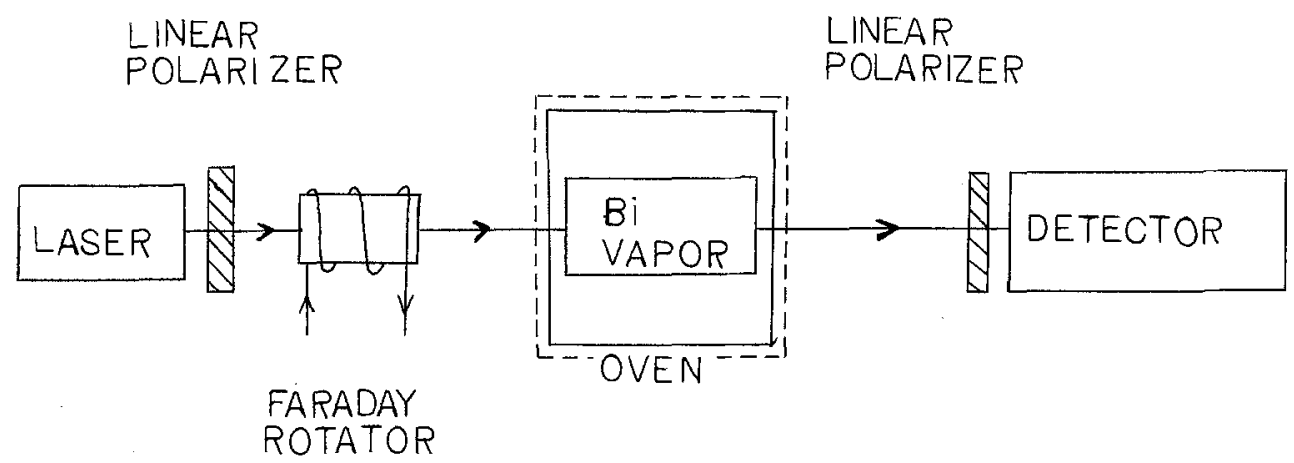

Figure 4. Apparatus to measure optical activity in Bismuth.

contains an interference term $\phi_{M}\left(\phi_{W}+\phi_{0}\right)$ which can be isolated by modulation of the magnetic field. The separation of $\phi_{0}$ from $\phi_{W}$, and the determination of $\phi_{W}$, is possible only by a study of the line shape of the transmission. The resonant line shape of $\phi_{W}$, and the magnitude and line shape of $\phi_{M}$, can be accurately calculated. The line shape of $\phi_{0}$ is unknown, but slowly varying.

The latest results from both experiments ${ }^{23}$

$$
\begin{array}{ll}
\phi_{W}=+(1.6 \pm 2.3) \times 10^{-8} & \text { (OXFORD) } \\
\phi_{W}=-(0.7 \pm 3.2) \times 10^{-8} & \text { (WASHINGTON) }
\end{array}
$$

are consistent with zero effect. These data are about 10 times smaller than the prediction of the Weinberg-Salam gauge mode $1^{23}$ but there is still some uncertainty about the accuracy of the atomic physics parts of the calculation of (El) W. The tentative conclusion from these data is that there is no large increase in $C_{1}$ (nucleus) $=Z C_{1 p}+N C_{i n}$ in bismuth, perhaps due to a predominantly isovector component $\left(C_{l p}=-C_{l n}\right)$, rather than an isoscalar component $\left(C_{1 p}=+C_{1 n}\right)$ in the weak

4) There is an experiment in progress by Chu, Commins and Conti at Berkeley (1976), studying the circular dichroism of thallium vapor $(\lambda=293 \mathrm{~nm})$ using a pulsed dye laser. The technique is similar to that of Bouchiat.

5) A series of experiments, using metastable hydrogen beams were initiated at Michigan by Lewis and Williams ${ }^{25}$ in 1976. Each of the experiments exploits level crossings in the $n=2$ shell, at which the parity mixture $F$ goes through a resonance as the magnetic field is varied. The parity mixtures are of order $F \simeq 10^{-10}$, and are equally sensitive to $C_{1}$ and $C_{2}$. The beam machine consists of a duoplasmatron ion source, followed by a cesium charge exchange cell and an interaction region containing an axial magnetic field, Fig. 5. A metastable $H$ and D source, built at Michigan by Clegg, Dunford and Williams, has been operating since 1976 with a $\mathrm{flux}$ of about $10^{+14} \mathrm{H*} / \mathrm{cm}^{2} \mathrm{sec}$.

One of the experiments involves an interaction region consisting only of static axial electric and magnetic fields $E_{Z}, B_{Z}$. A $2 S$ atom experiencing adiabatic entry into $B_{Z}$ but sudden entry into $E_{Z}$ has a $2 P^{2}$ component induced by the electric field, in addition to the $2 \mathrm{P}$ component induced by the weak interaction. Each of 
DUOPLA SMATRON

ION SOURCE

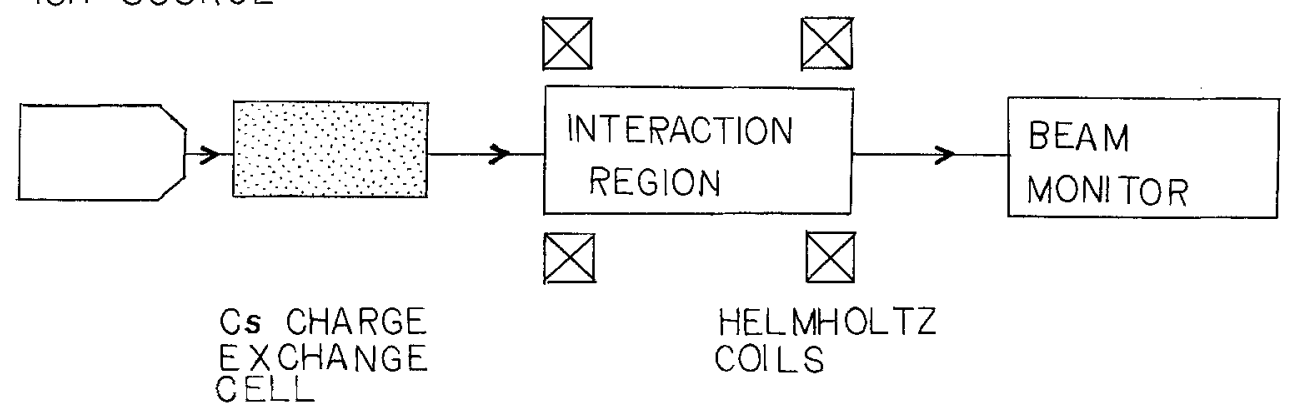

Figure 5. Metastable Hydrogen beam apparatus.

these $2 \mathrm{P}$ components gives a decay amplitude for Lyman- $\alpha$ emission $(2 \dot{P} \rightarrow 1 S$ ) which interfere in the decay rate of the beam. This experiment is analogous to the 'coherent regeneration' effect used to measure CP nonconservation in neutral $K$ mesons. The result is a dependence of the Lyman- $\alpha$ production on the scalar $E^{2}$ and on the psuedoscalar $\vec{E} \cdot \vec{B}$. The pseudoscalar terms can be identified in the detector output by modulation of $E$ and $B$. This experiment is in progress with a $100 \mathrm{keV}$ beam at Windsor, in collaboration with Drake, van Wijngaarden and Helbing. The sensitivity of this experiment is proportional to $E^{-1}$, and for $E=1 \mathrm{Vol} / \mathrm{cm}$ is about $10^{-8}$. (see Fig. 6)

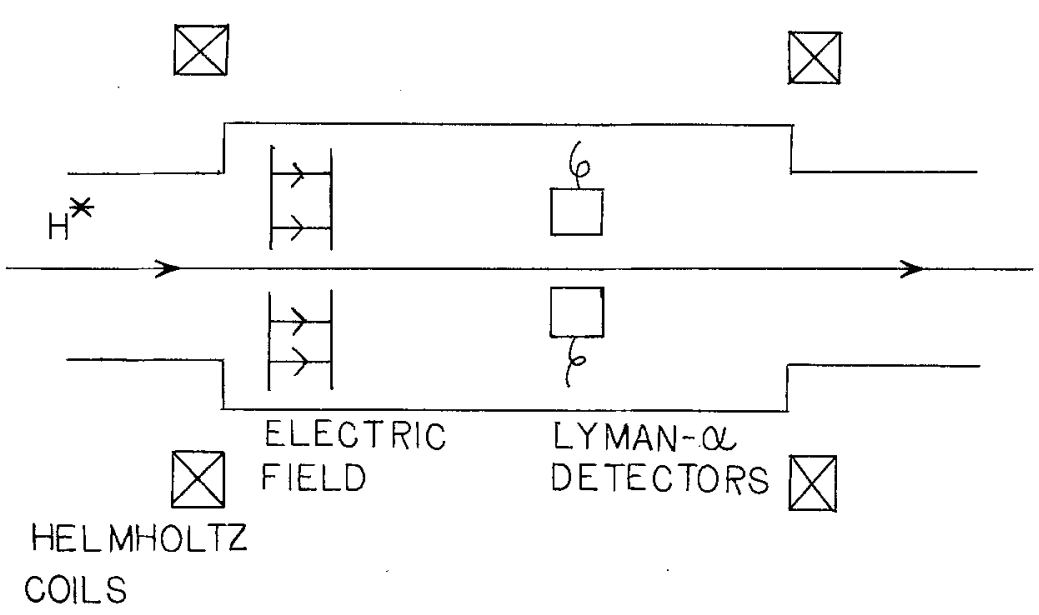

Figure 6. Interaction region for electric regeneration experiment. 
Another experiment in progress at Michigan involves a measurement of the rate of $2 S \rightarrow 3 S$ transitions $(\lambda=656 \mathrm{~nm})$ with a $\mathrm{CW}$ tunable dye laser, Fig. $7 \mathrm{a}$. The transition amplitude consists of three terms, the intrinsic magnetic dipole amplitude (MI), the Stark-induced electric dipole amplitude (EI) $S$ and the weakinduced electric dipole amplitude $(E I)_{W}$. These amplitudes depend on the polarization vector $\hat{\varepsilon}$, the propagation vector $\hat{k}$ and the electric and magnetic fields $E$, B. Since there are so many variables there are several different ways of building a 'handed' interaction region. We have elected to measure the psuedoscalar $\hat{E} \cdot \vec{E} \hat{E} \cdot \vec{B}$, arising from the interference of $(E I)_{S}$ and $(E I)_{W}$. The configuration of these vectors is shown in Fig. 7b. A detector downstream from the intersection of the laser and atomic beam has a rate proportional to $(\hat{\varepsilon} \cdot E)^{2}$, and a sensitivity proportional to $E^{-1}$. For $E=1$ volt $/ \mathrm{cm}$ the sensitivity is about $10^{-8}$ and the integration time is about one day.

A third experiment involving microwave transitions $2 S \rightarrow 2 S^{\prime}$ at $f \cong 1600 \mathrm{MHz}$ is also in progress at Michigan. ${ }^{26}$ it is described in more detail in the next section.

6) A microwave experiment with a metastable hydrogen beam is in progress at Yale by Hinds and Hughes $7(1977)$. They propose to measure the interference of $(M 1)$ and $(E I)_{W}$ in microwave transitions $2 S \rightarrow 2 S^{\prime}$. The scalar term is $(\mathrm{m} \times \mathrm{B})^{2}$ and the psuedoscalar term $(E \times m \cdot B)$, where $\varepsilon, m$ are the oscillatory electric and magnetic fields. The sensitivity is proportional to $|\varepsilon / \mathrm{m}|$, which they estimate can be made as large as 15 to enhance the PNC signal.

To summarise this survey, there is a vigorous competition underway to search for parity nonconserving effects of order $f$. The experiments using resonance fluorescence in cesium have not yet yielded data to this level of sensitivity, due primarily to the relatively large backoround of resonance radiation generated by collisions. The experiments based on optical activity have achieved a sensitivity to PNC terms 10 times smaller than predicted by the Weinberg-Salam model, without seeing any significant parity nonconservation. The conclusion, pending further work on the atomic theory, is that the growth in the 'weak charge' of nuclei with increasing atomic weight does not seem to occur.

\section{MICROWAVE EXPERIMENT AT MICHIGAN}

The rather superficial description of the various experiments reviewed in the previous section can best be counteracted by a more detailed treatment of one particular example, presently in progress at Michigan. 26 . While many of the details are specific to the hydrogen atom, the overall results typify similar problems in this field.

The hydrogen atom offers obvious advantages in searching for a PNC electronproton interaction. Copious sources are available, the atomic states are known to high precision $(\simeq 1 \mathrm{ppm})$ and the spectrum contains closely spaced 'parity doublets' with the same $J$ and opposite parity, like $2 S_{1 / 2}-2 P_{1 / 2}$. The ability to calculate everything is a major advantage in planning the experiment, as well as in analyzing results. Whether an effect is seen or not, the results can be unambiguously related to the weak interaction coupling constants. The major difficulty is the very small value of the parity mixture: for zero field, the mixture of $2 S-2 P$ states in the $F=1$ hyperfine state can be shown to be

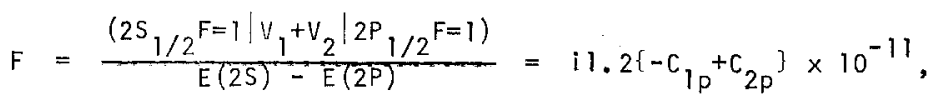

which seems hopeles sly small. 


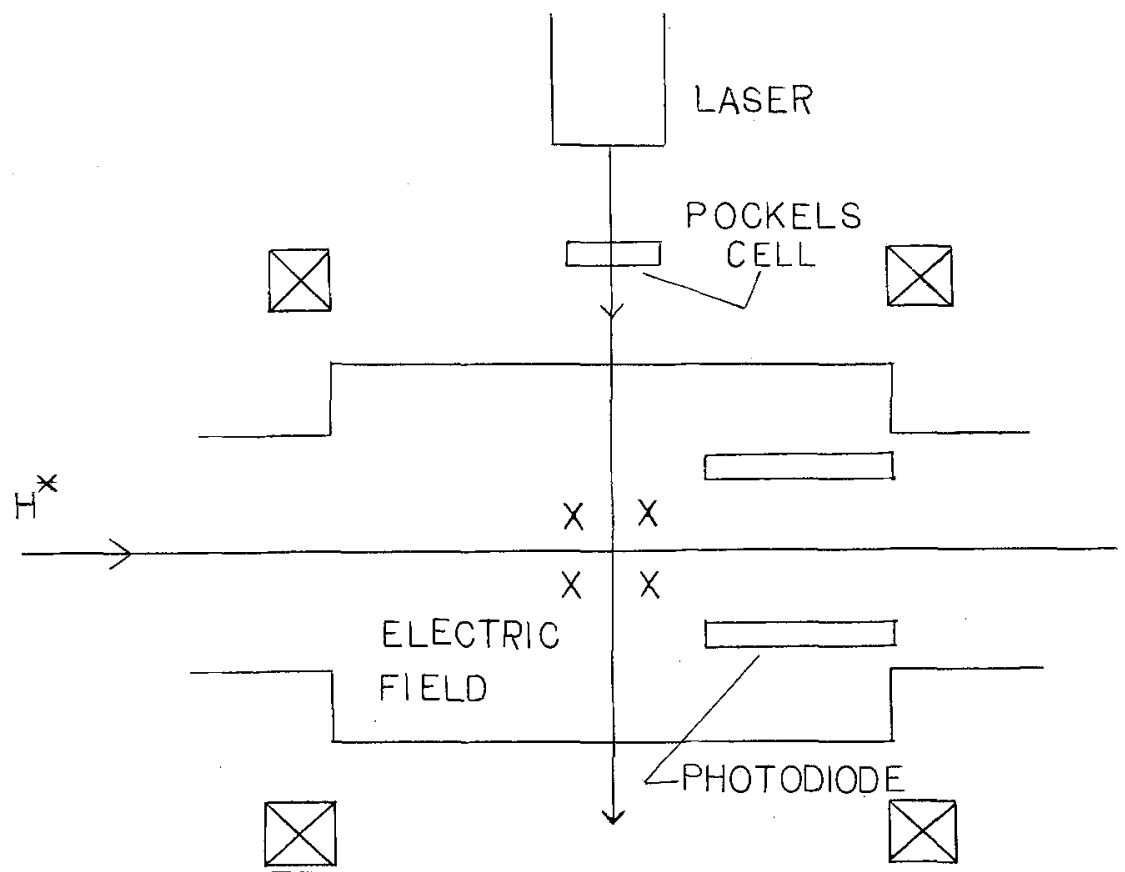

HELMHOLTZ

COILS

(a)

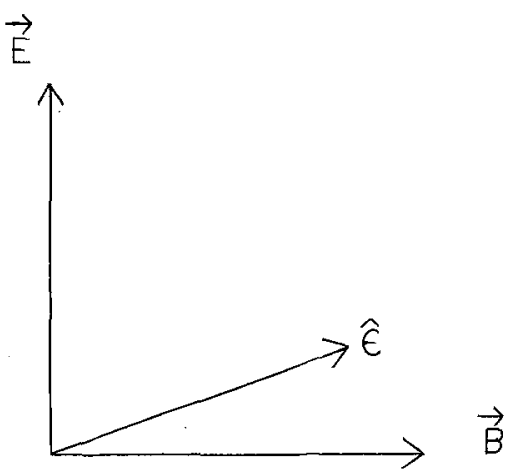

(b)

Figure 7. (a) Interaction region for Balmer-alpha absorption experiment.

(b) Diagram showing orientation of vectors in interaction region. 
The small energy difference, which is in the microwave frequency regime, implies that the states can be strongly perturbed by external fields. In Fig. 8 we show the energy level diagram versus magnetic field for $n=2$ hydrogen. At certain field strengths levels may 'cross', such as the $\beta$-e states at 575 Gauss. The behavior of the atom in the vicinity of this field is governed by the damping constants of these levels $\left(\Gamma_{2 S} \approx 0, \Gamma_{2} \approx 100 \mathrm{MHz}\right)$; at the 'level crossing' the real part of the energy difference goes through zero but the imaginary part $-i \Gamma / 2$ remains approximately constant. Consequently the energy denominator in Eq. 17 is replaced by $\Delta \mathrm{E} \rightarrow+\mathrm{i} \mathrm{T}_{2 \mathrm{p}} / 2$ and the parity mixture is increased by a factor $1057 \mathrm{MHz} / 50 \mathrm{MHz}=21$ relative to zero field. Of equal importance is the flexibility of being able to sweep the magnetic field through this resonance, producing a variable parity mixture with known line shape. For these reasons, parity experiments in hydrogen should be done at the level crossings. An added advantage of the $575 \mathrm{G}$ crossing is that it selects the contributions of $V_{2}$ alone, giving a parity mixture at crossing

$$
F=\frac{\left(2 S_{1 / 2}, \beta\left|V_{1}+V_{2}\right| 2 P_{1 / 2}, e\right)}{i \Gamma_{2 P} / 2}=5.0 c_{2 p} \times 10^{-10}
$$

which is somewhat bigger. The cancellation of $V_{1}$ at this field is essentially a fortuitous accident of the pure coulomb field.

The use of microwave transitions is indicated by the large aperture and strong fields of a cavity, giving a transition rate equal to a significant fraction of the entire flux of metastable atoms from the ion source. The experiment is designed to observe the stimulated emission line $\alpha_{0} \rightarrow \beta_{0}$ at about $1600 \mathrm{MHz}$ (see Fig. 9). These transitions can be detected by first removing the $\beta$-states with an electric quenching field ( $\approx 10 \mathrm{~V} / \mathrm{cm}$ transverse to $\mathrm{B} \simeq 575 \mathrm{G}$ ), and then re-populating the $\beta_{0}$ state by microwave transitions. The electric quenching field mixes $\beta$ and e states, yielding spontaneous emission of Lyman- $\alpha$, without appreciable attenuation of the $\alpha$ states. The regenerated $\beta_{0}$ atoms are sufficiently long-lived to pass out of the cavity into another electric quenching field, where they are detected by the appearance of Lyman- $\alpha$ radiation. Thus the beam is first prepared in the a states by removal of $\beta^{\prime} s$, and then pumped down to the $\beta_{0}$ state, where it is again quenched for detection of the $\beta_{0}$ atoms; see Fig. 10.

The transition rate $\alpha_{0} \rightarrow \beta_{0}$ can be made into a sensitive test of parity conservation by giving the interaction region (cavity) a handedness, which can then be reversed. This could be done in a variety of ways, since we can apply both static fields $(E, B)$ and microwave fields $(\varepsilon, b)$ to the $\alpha_{0}$ beam. In the presence of perturbations, the $\alpha_{0} \rightarrow \beta_{0}$ transition can be generated either by the magnetic field (b) coupling to the magnetic dipole moment, or by the electric field $(\varepsilon)$ coupling to the weak-induced and Stark-induced electric dipole moments. In order to enhance the PNC amplitude, we eliminate the magnetic transition by choosing a cavity mode $\left(\mathrm{TM}_{010}\right)$ which has only an electric field along the beam. In this mode, the $P C$ transition now requires an electrostatic field ( $E_{x}$ ) transverse to the magnetic field $\left(B_{z}\right)$. This additional field, which is produced by a fine grid of wires strung through the cavity, gives added flexibility in adjusting the strength of the $P C$ transition, as well as an additional 'signature' for the PNC effect, which will be odd in $E_{X}$. An upper limit to the strength of $\varepsilon$ is set by the requirement that the $\alpha_{0}$ state not be attenuated by more than about $10 \%$ in passing through the cavity. The diagrams. for these processes are shown in Fig. 11 as stimulated microwave emission to the short-lived $e_{+}$, e e states, which spontaneously decay to the ground state inside the cavity. Since the projection of the total angular momentum along the magnetic field $\left(m_{F}\right)$ is a good quanturn number, transitions $\alpha_{0} \rightarrow e_{+}$can only be generated by transverse components of the $m i$ crowave field $\left(\varepsilon_{x}\right.$ or $\left.\varepsilon_{y}\right)$, which change $m_{F}$ by \pm 1 units. The transitions $\alpha_{0} \rightarrow e_{0}$ are generated by $\varepsilon_{z}$, which preserves $m_{F}$. 


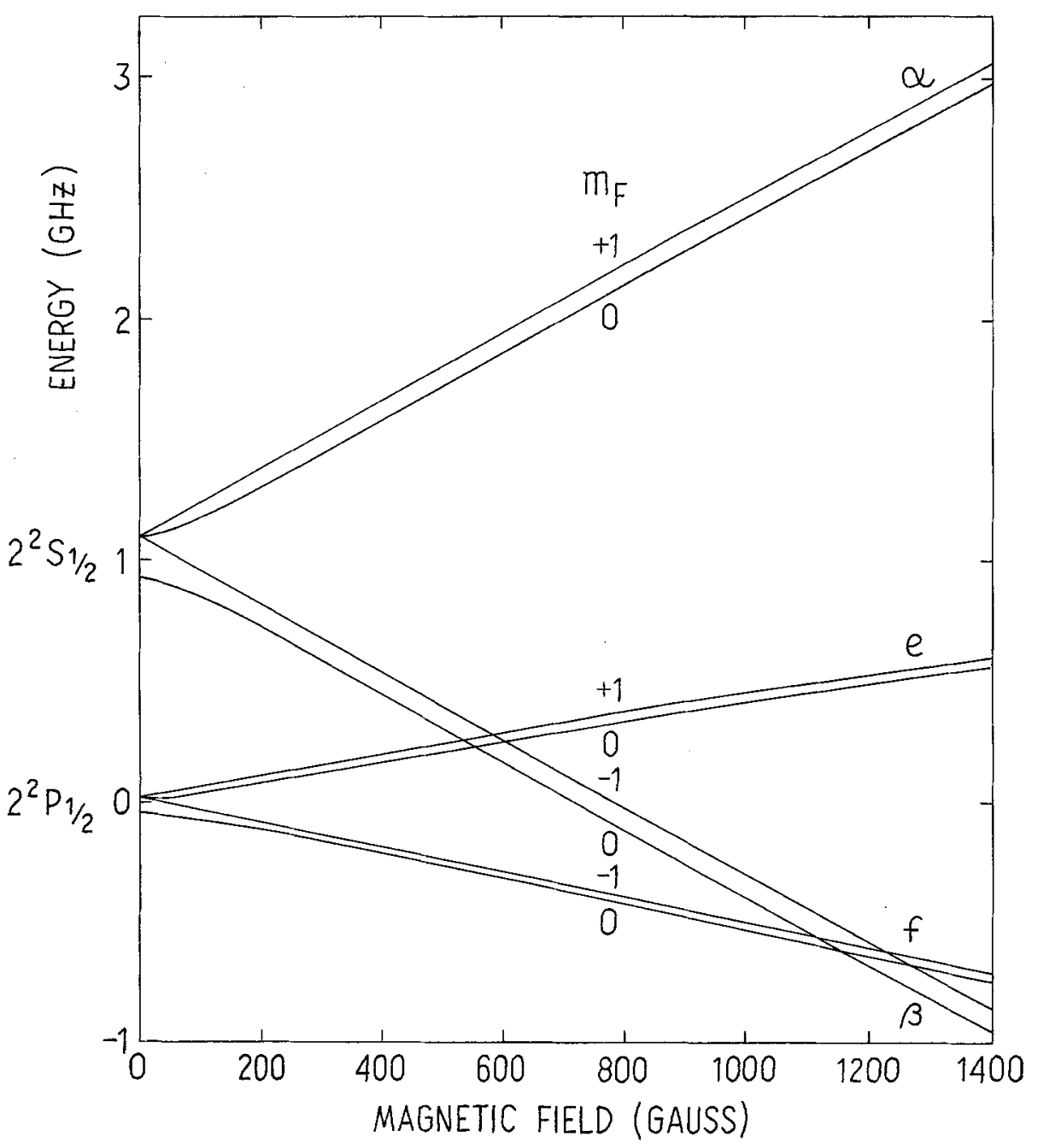

Figure 8. Energy levels of Hydrogen versus magnetic field, showing the level crossings of $2 S-2 P$ states. The levels are labeled using Lamb's notation, with the hyperfine sublevels denoted by subscript $m_{F}$. 


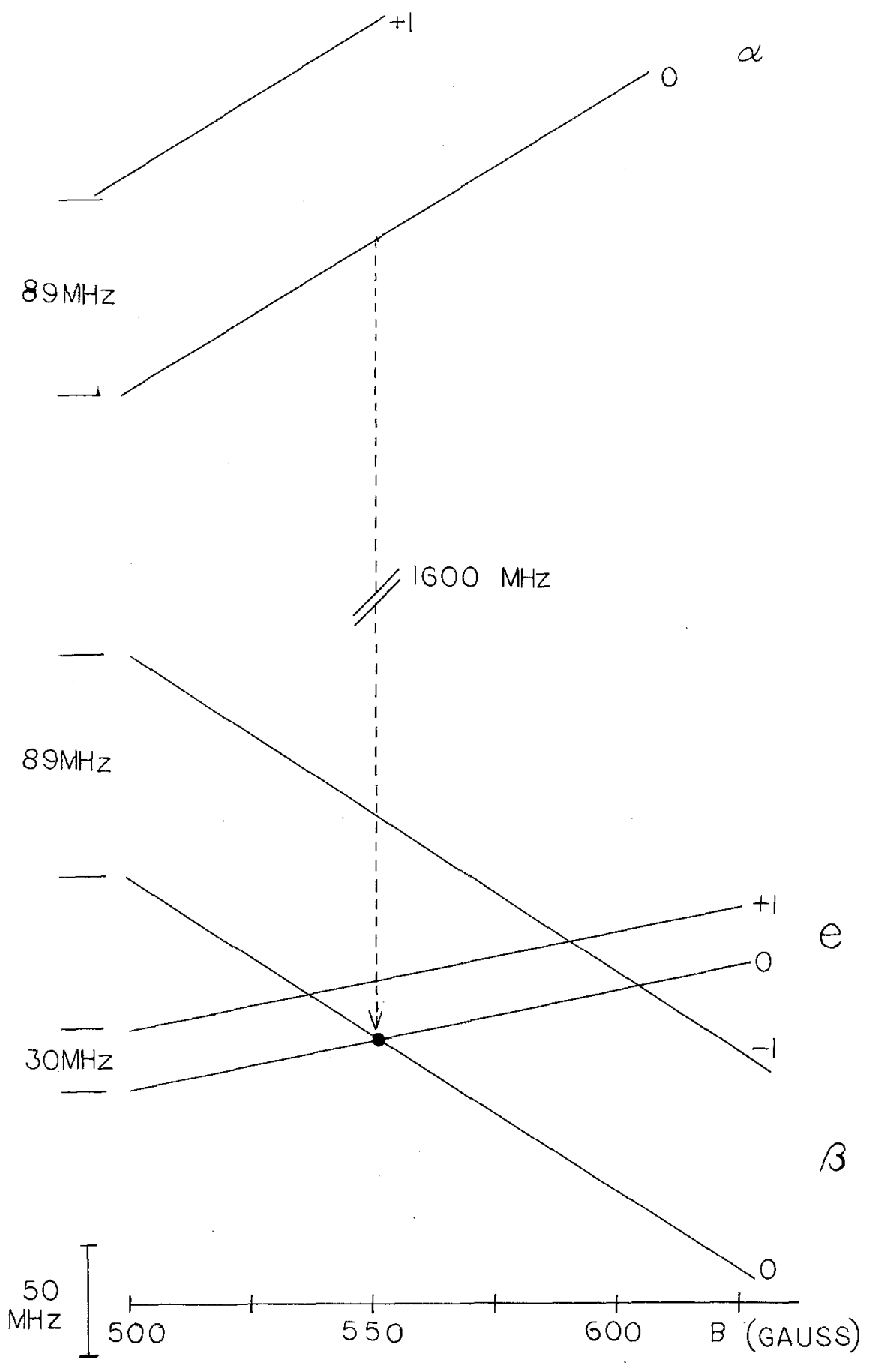

Figure 9. Microwave transition 0 at level crossing. 


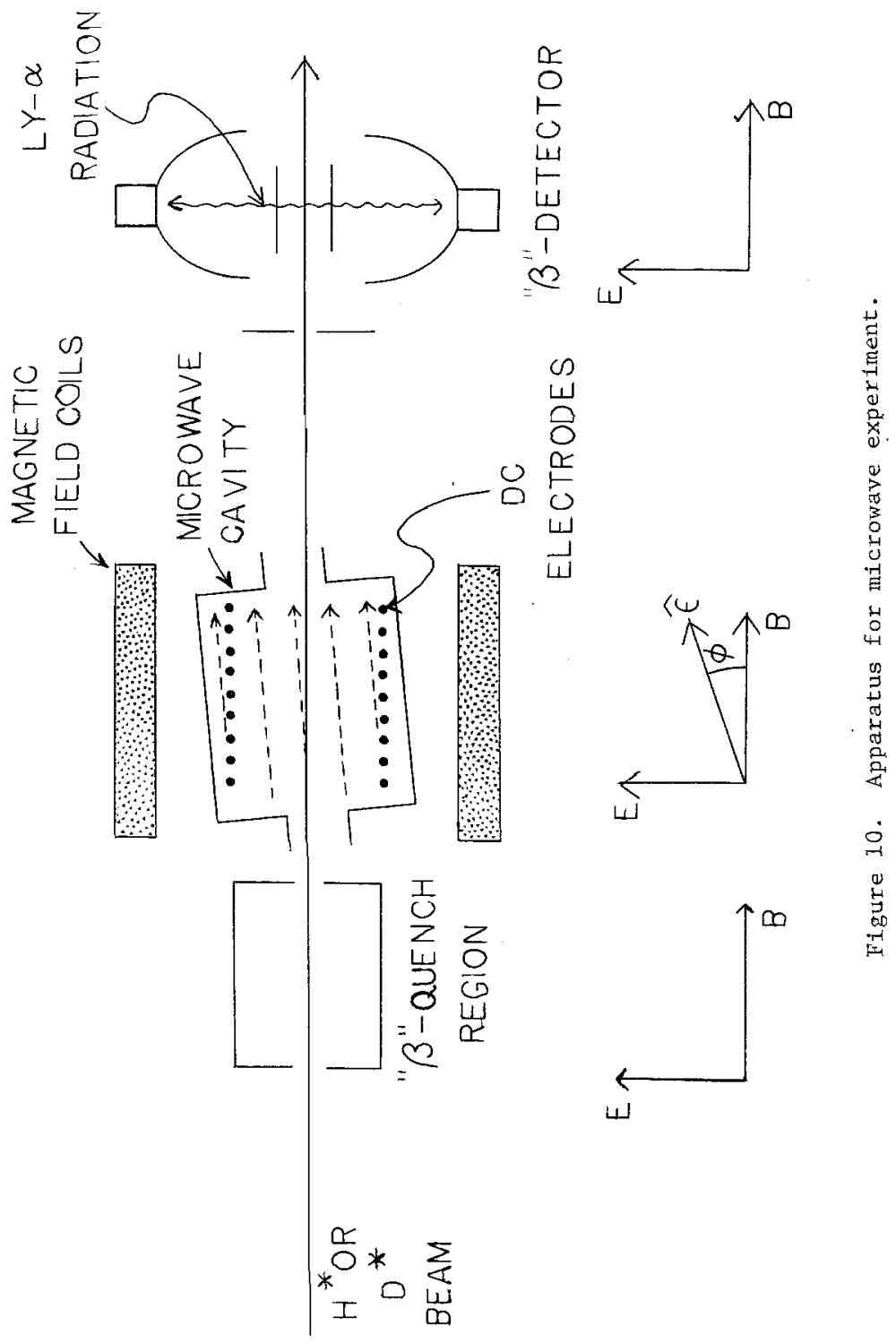


The transitions $\alpha_{0} \rightarrow \beta_{0}$ recorded by the detector downstream arise through the higher order process in which the intermediate e-states are mixed with $\beta-$ states by the static electric field and by the weak interactions, (see Fig. 12). Since we are at (or near) the level crossing of $\beta$-e states, a static perturbation causes a resonant mixing of these states. Selection rules on the good quantum number $m_{F}$ dictate which states are coupled by the different perturbations. If . the static electric field is applied in the $x$-direction, transverse to the magnetic field and the atomic beam, then the PC diagram (11a) is dependent only on the strength of $\varepsilon_{x}$, the transverse component of the microwave electric field. Each of these perturbations can change $m_{F}$ by \pm 1 , giving a transition from $\alpha_{0} \rightarrow \beta_{0}$ with $\Delta m_{F}=0$. The PNC diagram (lib), on the other hand, depends only on $\varepsilon_{z}$ since the weak interaction $V$ preserves $\pi_{F}$. By inspection of these diagrams, it is now evident that the $P C$ transition rate is proportional to the scalar $\varepsilon_{x}^{2} E_{x}^{2}$, coming from the square of the amplitude of diagram lla, while the PNC term in the transition rate is proportional to the pseudoscalar $E_{X} E_{X} \varepsilon_{z} B_{z}$, coming from the interference of the amplitudes from these two diagrams. Clearly, a small PNC signal can be detected from background by a modulation scheme which reverses $E_{x}, B_{z}$ and $\varepsilon_{x} \cdot$

The sensitivity to a small PNC amplitude can be further increased by suppressing diagram a), without diminishing diagram b). One way of accomplishing this is to adjust the ratio $\varepsilon_{X} / \varepsilon_{z}$ by setting the cavity axis at a small angle $\left(5^{\circ}\right)$ relative to the magnetic field (see Fig. 10). An additional gain in sensitivity arises naturally in this configuration due to the small size of the electric dipole matrix element $\left(e_{+}|x| \alpha_{0}\right)$ compared with $\left(e_{0}|x| \alpha_{0}\right)$. The latter is of order $a_{0}$, since it does not involve flipping either the electron or proton spin $\left(\Delta m_{J}=\Delta m_{1}=0\right)$. The former is of order $(1 / 10) a_{0}$, since it requires flipping both spins $(\Delta m=+1, \Delta m=-1)$. This is possible only through the hyperfine coupling of $I$ and $J$, which is weak compared with the Zeeman energy and the fine structure.

Combining all these factors, the predicted ratio of PNC and PC terms in an applied field of about $1 \mathrm{~V} / \mathrm{cm}$ is $2.5 \mathrm{C}_{2} \times 10^{-6}$, and the integration time for observing this signal in a background of statistical (shot) noise is about 30 minutes. Therefore, by careful choice of the configuration, and accurate alignment of the beam and the various perturbing fields, the contributions of weak interactions can be made as large as a feh ppm. It is reasonable to expect to measure in this way $C_{2}$ in hydrogen and $C_{2 p}+C_{2 n}$ in deuterium. Similar experiments at the other level crossings permit the determination of $C_{1}$ and $c_{3}$ to order unity.

\section{E. CONCLUSIONS AND SUMMARY}

The results of experiments completed at this date are either inconclusive or null, which should leave a certain skepticism about the existence of such weak interactions and our ability to detect them. This should be balanced however by the technical advances which have been made sensitive parity experiments, and by the general success of gauge theories of weak interactions and electromagnetism. It seems quite likely that experiments with atoms -- whether hydrogen beams or heavy metal vapors or something different - will soon be able to determine the constants, $C_{1}, C_{2}$ and $C_{3}$. The values of these constants in the future will be, as $C_{V}$ and $C_{A}$ have been in the past, a major input to determination of the structure of weak interactions. Small deviations from Laporte's rule in simple atoms may eventually lead to an understanding at the microscopic level of long standing questions about the origin of handedness in organic mole cules and in biochemistry. It should be noted that many of the techniques discussed in this review can be naturally extended to molecular physics as well. 


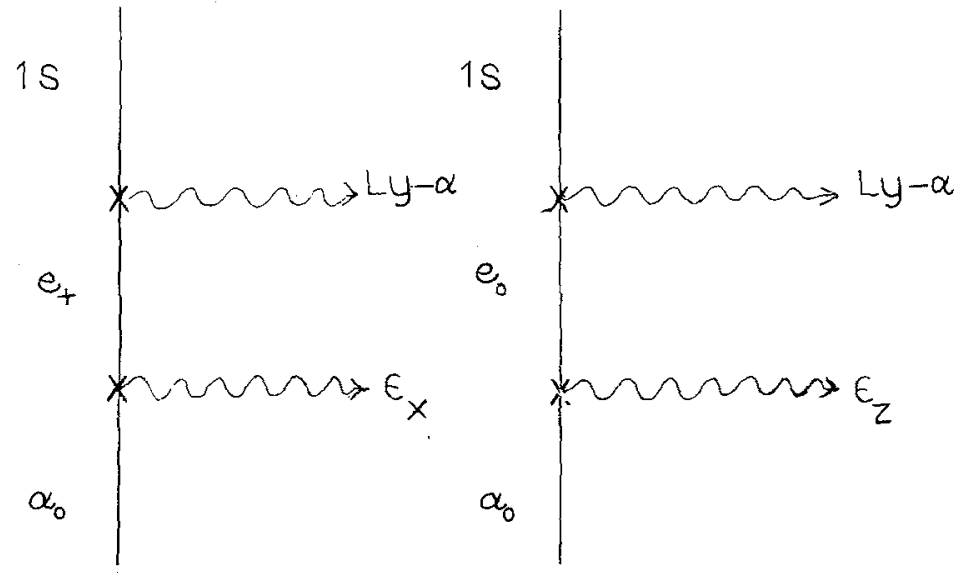

Figure 11. Diagrams for the attenuation of alpha states by microwave transitions e.

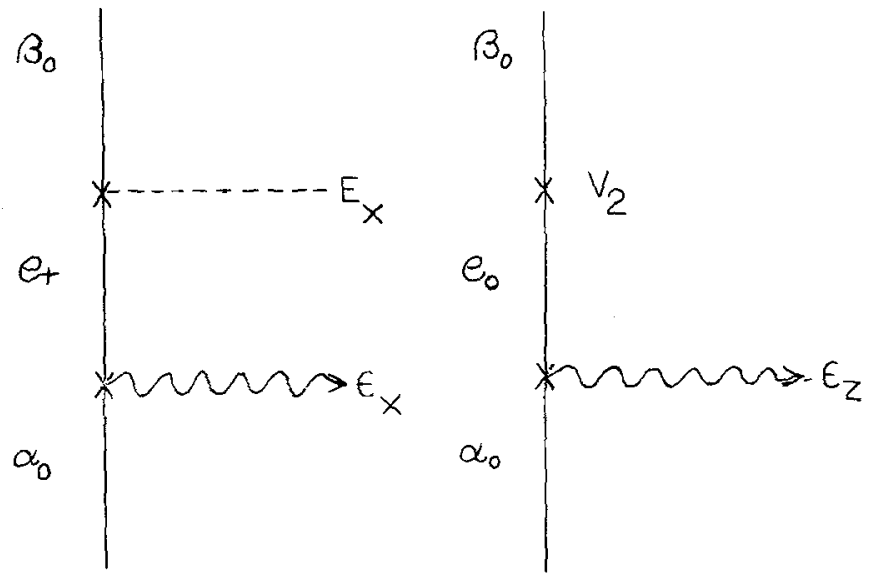

Figure 12. Diagrams for the microwave transitions $00^{\circ}$ 


\section{$\exists$ ЭUTAИ 2ЯОНЯA Tว 37939 ИOISA VVI ! YЯT JMMY2}

\section{NATURE ABHORS PERFECT INVERSION SYMMETRY!}

Figure 13. Do-it-yourself parity experiment: are these mirror images? (Hold this up to a mirror!)

A suitable motto for this subject is shown in Fíg. 13, illustrating a weakly broken mirror symmetry: all of the letters but one have been reversed. Held up to a mirror, the faulty letter is much more easily found: This illustrates the bias of our brain, and explains why data taking on a parity experiment should be left to a computer.

\section{F. Acknowledgement.}

It is a pleasure to acknowledge the collaborative contributions of $\mathrm{W}$. L. Williams throughout the course of the work on experiments in hydrogen, and in the preparation of this review.

\section{REFERENCES}

1. O. Laporte, Zs fur Physik 23135 (1924).

2. E. Wigner, Zs fur Physik 43624 (1927).

3. T. D. Lee and C. N. Yang, Phys, Rev. 104254 (1956). For further details see Wu and Moszkowski "Beta Decay" Interscience (1966).

4. G. Feinberg, "Atomic Physics", p 1-14, Benjamin NY (1969); P. G. H. Sandars, "Atomic Physics", p 71-92, Plenum Press NY (1975).

5. S. Weinberg, Rev. Mod. Phys., 46255 (1974).

6. Y. Zeldovich JETP 36964 (1959); F. C. Michel, Phys. Rev. 138 B408 (1965).

7. F. J. Hasert et al, Phys. Letters 46B 138 (1973); A. Benvenuti et a1, Phys. Rev. Letters 32800 (1974); S. J. Barish et al, Phys. Rev. Letters $33448(1974)$.

8. The latest results have been summarized at a Neutrino Conference, Aachen, June 1976.

9. Aachen - Padua collaboration; see ref. 7.

10. C. Bouchiat, Phys. Letters 57B 184 (1975); E. A. Hinds, C. A. Loving and P. G. H. Sanders, Phys. Letters $62 B 97$ (1976).

11. Y. Azimov et al, JETP $6717(197 \overline{4})$; M. A. Bouchiat and C. C. Bouchiat, Phys. Letters 483 111 (1974); G. Feinberg and M. Y. Chen, Phys, Rev. D10 190 (1976).

12. R. N. Cahn and G. Kane, Univ, of Michigan HE 77-1, to be published.

13. R. W. Dunford, R. R. Lewis and W. L. Williams, "Parity Nonconservation in the Hydrogen. AtomII," submitted to Phys. Rev. D.

14. M. A. Bouchiat and C. C. Bouchiat, J. Physique 35899 (1974); J. Physique 36 493 (1975).

15. M. A. Bouchiat and L. Pottier, J. Physique 36189 (1975); J. Physique 37 79L (1976); Phys. Letters 62B 327 (1976). 
16. For a general review of this subject see F. Boehm, "Hyperfine Structure and Nuclear Radiations" North Holland (1976) p 279.

17. R. R. Lewis, "Hyperfine Structure and Nuclear Radiations", North Holland 1967 , p. 318; Phys. Rev. 163935 (1967).

18. A. N. Moskalev, ZhETF Pis. Red. 19229 (1974) (JETP Letters 19141 1974);

Ya. I. Azimov et a1, ZhETF 67 17 (1974) (JETP 408 1975).

19. I. B. Khriplovich, ZhETF Pis. Red. 20686 (1974) (JETP Letters 20315 (1974)).

20. V. A. Alekseev et al, Usp. Fiz. Nauk 118409 (1976); (Sov. Phys. Usp. $19220(1976))$.

21. P. G. H. Sandars, in "Atomic Physics IV" ed G. zuPutlitz, Plenum Press NY (1975).

22. D. C. Soreide et al, Phys. Rev. Letters 36352 (1976).

23. P. E. G. Baird et a1, Nature 264528 (19776; P. G. H. Sandars and N. Fortson, BAPS 22524 (1977), invited addresses to APS, May 1977.

24. S. Chu, B. Commins and R. Conti, Fifth International Conference on Atomic Physics, Berkeley (1976).

25. R. R. Lewis and W. L. Williams, Phys. Letters 59B 70 (1975).

26. R. W. Dunford et al "Parity Nonconservation in the Hydrogen Atom III" submitted to Phys. Rev. D (1977).

27. E. A. Hinds and V. W. Hughes, Phys. Letters $67 \mathrm{~B} 487$ (1977).

\section{DISCUSSION}

R. S. Raghavan: Could you please elaborate on the implications of the negative result of the Oxford-Washington experiments to weak interaction theory, in particular the WeinbergSalam theory.

R. R. Lewis: The bismuth results, pending re-calculation of the atomic part of the theory, appear to contradict the Weinberg-Salam model, which predicts a large isoscalar contribution $C_{1 p}=C_{1 n}$. The simplest implication would be a dominant isovector theory with $C_{1 p}=-C_{1, n}$ In gauge theory this requires extra leptons, or extra intermediate mesons, with large masses.

F. P. Calaprice: Would you comment on the controversy over estimates of the effect of configuration mixing - in particular the estimates of Meshkov, et al.

R. R. Lewis: Atomic theorists do not yet agree on the calculation of $R_{s}(o) R_{p}{ }^{\prime}(o)$ in bismuth. There are several published calculations with fairly small $(\sim 20 \%)$ differences, and a published criticism of the handling of configuration mixing of D-states. Sandars outlined a new method of calculation of the Washington APS meeting (1977) and promised results in about one month.

M. Leventhal: At the 575 Gauss level-crossing in hydrogen, your experiment will be very sensitive to stray electric fields, slight misalignments, etc. Will it be possible to control systematic effects which seem to me to be larger than the effect you are looking for?

R. R. Lewis: Yes, the experiment is very sensitive to stray electric fields. Even with stray fields of millivolt $/ \mathrm{cm}$, and alignment errors of $10^{-3}$ radian, the systematic effects will dominate the parity signal. We have four separate reversals to beat down their effect: reversal of $\vec{E}$, reversal of $\vec{B}$, change of polarization and magnetic lineshape. Only time will tell whether this is good enough.

J. M. Daniels: In a Lamb Shift Polarized Ion Source at 575 Gauss, mixing occurs and the metastable states decay and are removed from the beam in a length of a few millimeters. Could you comiment on this? 
R. R. Lewis: Yes, the $\beta$ states at 575 Gauss can be quenched by an electric field of a few volts $/ \mathrm{cm}$. A stray field, or a motional $\vec{v} \times \vec{B} / c$ field, will do this. The beams machine must be kept free from insulators, must be gold-plated, etc. to avoid this. We have operated a beam for over a year and have satisfactorily passed a $\beta$ beam through the interaction region and magnetic field.

S. G. Cohen: The motivation for these experiments is the belief that the neutral weak interaction does not conserve parity. What are the chances of this being investigated directly in a high energy experiment?

R. R. Lewis: The weak interaction amplitude rises linearly with lab energy and therefore should produce much bigger effects in high energy $(e, p)$ elastic scattering. There is already one experiment at SLAC showing a null effect at about $10^{-3}$. There is definitely a "competition" between high energy physics, where the effect is larger but the precision is lower, and low energy physics, where the reverse is true.

H. J. Kluge: Let me briefly comment. At the university of Mainz we are preparing an experiment in order to test the parity violation by neutral currents in the electron-nucleon scattering. We search for an asymmetry in the cross section for the scattering of electrons polarized parallel or antiparallel to the direction of their momentum. An asymmetry effect of the order of $10^{-4}$ is expected for $300 \mathrm{MeV}$ electrons provided that the Weinberg-Salam theory is right.

L. Grodzins: Please comment on early limits on parity violation in atomic systems, particularly on the work of Bradley and Wall.

R. R. Lewis: There are earlier experiments on optical activity in $\mathrm{O}_{2}$ by Bradley and Wall, and circular dichroism in $\mathrm{Pb}$ by Poppe, with null results at a level of about $10^{-3}$ or $10^{-4}$ (raw asymmetry). These systems have larger MI matrix elements than Cs and are much less sensitive to weak interactions. 